\title{
Behavior of a hollow core photonic crystal fiber under high radial pressure for downhole application
}

J. Sadeghi, H. Latifi, J. L. Santos, Z. Chenari, and F. Ziaee

Citation: Appl. Phys. Lett. 104, 071910 (2014);

View online: https://doi.org/10.1063/1.4866334

View Table of Contents: http://aip.scitation.org/toc/apl/104/7

Published by the American Institute of Physics

\section{Articles you may be interested in}

Measurement and control of pressure driven flows in microfluidic devices using an optofluidic flow sensor

Biomicrofluidics 8, 054123 (2014); 10.1063/1.4900523

Whispering gallery mode selection in optical bottle microresonators

Applied Physics Letters 100, 081108 (2012); 10.1063/1.3688601

Temperature-insensitive photonic crystal fiber interferometer for absolute strain sensing

Applied Physics Letters 91, 091109 (2007); 10.1063/1.2775326

Mach-Zehnder interferometric photonic crystal fiber for low acoustic frequency detections

Applied Physics Letters 108, 041912 (2016); 10.1063/1.4940983

\section{Scilight}

Sharp, quick summaries illuminating the latest physics research

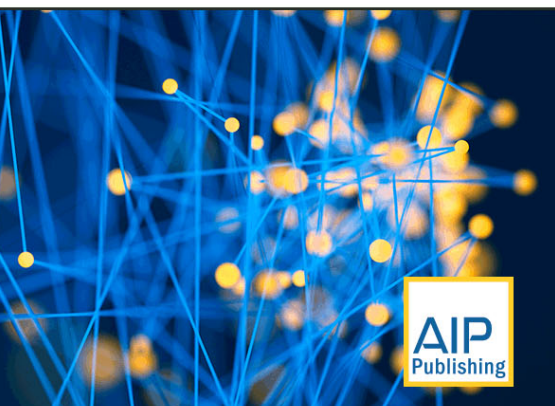




\title{
Behavior of a hollow core photonic crystal fiber under high radial pressure for downhole application
}

\author{
J. Sadeghi, ${ }^{1, a)}$ H. Latifi, ${ }^{1,2, b)}$ J. L. Santos, ${ }^{3,4, c)}$ Z. Chenari, ${ }^{1}$ and F. Ziaee ${ }^{1}$ \\ ${ }^{1}$ Laser and Plasma Research Institute, Shahid Beheshti University, 1983963113 Tehran, Iran \\ ${ }^{2}$ Department of Physics, Shahid Beheshti University, Evin, 1983963113 Tehran, Iran \\ ${ }^{3}$ INESC Porto-Instituto de Engenharia de Sistemas e Computadores do Porto, Rua do Campo Alegre, \\ 687, 4169-007 Porto, Portugal \\ ${ }^{4}$ Departamento de Física, da Faculdade de Ciências, da Universidade do Porto, Rua do Campo Alegre, \\ 687, 4169-007 Porto, Portugal
}

(Received 10 November 2013; accepted 6 February 2014; published online 20 February 2014)

\begin{abstract}
Pressure fiber sensors play an important role in downhole high pressure measurements to withstand long term operation. The purpose of this paper is to present an application of hollow core photonic crystal fiber (HC-PCF) as a high pressure sensor head for downhole application based on dispersion variation. We used a high pressure stainless steel unit to exert pressure on the sensor. The experimental results show that different wavelengths based on sagnac loop interferometer have additive sensitivities from $5 \times 10^{-5} \mathrm{~nm} / \mathrm{psi}$ at $1480 \mathrm{~nm}$ to $1.3 \times 10^{-3} \mathrm{~nm} / \mathrm{psi}$ at $1680 \mathrm{~nm}$. We developed a simulation to understand the reason for difference in sensitivity of wavelengths and also the relationship between deformation of HC-PCF and dispersion variation under pressure. For this purpose, by using the finite element method, we investigated the effect of structural variation of HC-PCF on spectral transformation of two linear polarizations under $1000 \mathrm{psi}$ pressure. The simulation and experimental results show exponential decay behavior of dispersion variation from $-3.4 \times 10^{-6} 1 / \mathrm{psi}$ to $-1.3 \times 10^{-6} 1 / \mathrm{psi}$ and from $-5 \times 10^{-6} 1 / \mathrm{psi}$ to $-1.8 \times 10^{-6} 1 / \mathrm{psi}$, respectively, which were in a good accordance with each other. ( 2014 AIP Publishing LLC.

[http://dx.doi.org/10.1063/1.4866334]
\end{abstract}

Recently, photonic crystal fibers (PCFs) have received a lot of attention due to their extensive potential applications. The new class of photonic crystal fibers with "holey" cladding and hollow core structure, with photonic band gap (PBG) guidance, has interesting properties such as low Rayleigh scattering, low transmission loss, low nonlinearity, band gap edge property, and surface modes compared to what happens in conventional waveguides. ${ }^{1-5}$ There are also many different kinds of hollow core PCFs (HC-PCFs) with different sensing applications such as fluorescence sensing, ${ }^{6}$ strain-temperature sensing, ${ }^{7,8}$ and acoustic pressure sensing. ${ }^{9}$

The polarization maintaining HC-PCFs was reported as an important subgroup of birefringence optical fibers which is investigated in the polarization properties of $\mathrm{HC}-\mathrm{PCF}$ with residual core ellipticity. ${ }^{10-12}$ In this paper, we have experimentally demonstrated the behavior of dispersion and sensitivity of one section of HC-PCF to the radial pressure. To understand the impact of radial structural deformation on birefringence changes, we have investigated the dispersion variation and spectral transformation of modes in HC-PCF under radial pressure by using the finite element method (FEM).

Figure 1(a) shows a scanning electron microscope (SEM) micrograph of special 7-cell HC-PCF including three different circular regions. These regions are irregular dodecahedron air core, honey comb inner cladding, and full silica outer cladding. The honey comb inner cladding has two distinctive parts. One of them is an innermost ring which

\footnotetext{
${ }^{a)}$ Electronic mail: j_sadeghi@sbu.ac.ir

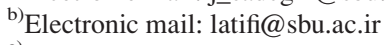

${ }^{c)}$ Electronic mail: josantos@fc.up.pt
}

contains two cell types with pentagon and hexagon faces and the other is formed of five rings of similar hexagons. As most of the light intensity is confined to the core and the nearest layers, we mostly focused on the variation of this region under radial pressure.

The cross section of HC-PCF used in our simulations and experiment is illustrated in Figs. 1(b) and 1(c), where the slow and fast diameters $\left(D_{s}\right.$ and $\left.D_{f}\right)$ of the central air core are $16.1 \mu \mathrm{m}$ and $15.3 \mu \mathrm{m}$, respectively. The rest of parameters are pitch $(\Lambda=4.12 \mu \mathrm{m})$, hole size $(\mathrm{d}=4.1 \mu \mathrm{m})$, length of the walls $\left(l_{1}=4.8 \mu \mathrm{m}, l_{2}=2.75 \mu \mathrm{m}, l_{3}=1.92 \mu \mathrm{m}\right)$, thickness of the walls $\left(\mathrm{t}_{\mathrm{w}}=190 \mathrm{~nm}, \mathrm{t}_{\mathrm{h}}=75 \mathrm{~nm}, \mathrm{t}_{\mathrm{p}}=155 \mathrm{~nm}\right)$, and angle at the innermost nodal points $\left(\theta_{1}=150^{\circ}, \theta_{2}=111^{\circ}, \theta_{3}=99^{\circ}\right)$.

In order to measure the sensitivity of the wavelengths to pressure $(\Delta \lambda / \Delta \mathrm{P})$ and the group birefringence of the HCPBGF in different polarization states, we utilized the sagnac loop interferometer. In the polarization maintaining fibers, the relationship between fiber length $(L)$, operation wavelength $(\lambda)$, experimental group birefringence $\left(B_{g \text {-exp }}\right)$, and the wavelength spacing of the peaks $(\Delta \lambda)$ in the sagnac loop interferometer can be written as

$$
B_{g-\exp }=\frac{\lambda^{2}}{L \Delta \lambda} .
$$

The experimental configuration is shown in Fig. 2(a), based on the sagnac loop interferometer. This loop was composed of a broadband light source (SLD-1560 nm), a conventional 3-dB coupler, an isolator, an optical spectrum analyzer (OSA-Agilent-86142B with a maximum resolution of $10 \mathrm{pm}$ ) a three plate polarization controller (PC), and a segment of 


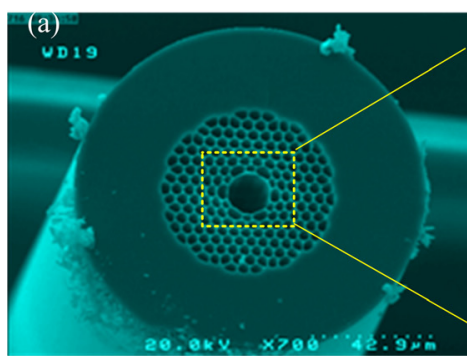

(b)

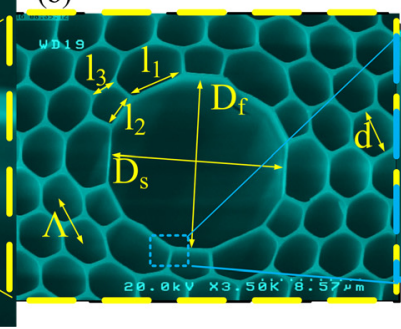

(c)

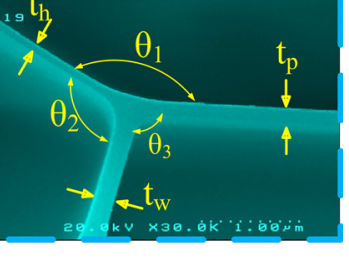

FIG. 1. (a) The SEM micrograph of HC-1550-NG 36. (b) and (c) The geometrical parameters of this HC-PCF.
HC-PCF with length of $30 \mathrm{~cm}$ as a sensing head. The HCPCF undergoes radial pressure by injecting water with a high pressure stainless steel unit. ${ }^{13}$

Finally, the sagnac loop interferometer causes two polarization modes which propagate with different phase and group velocities and interfere at the output port. The pressure was measured by using a digital pressure gauge with \pm 2 psi pressure accuracy. The experimental results showed that the pressure sensitivity of the investigated HC-PBG fiber was in

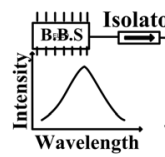

(a)

(b)
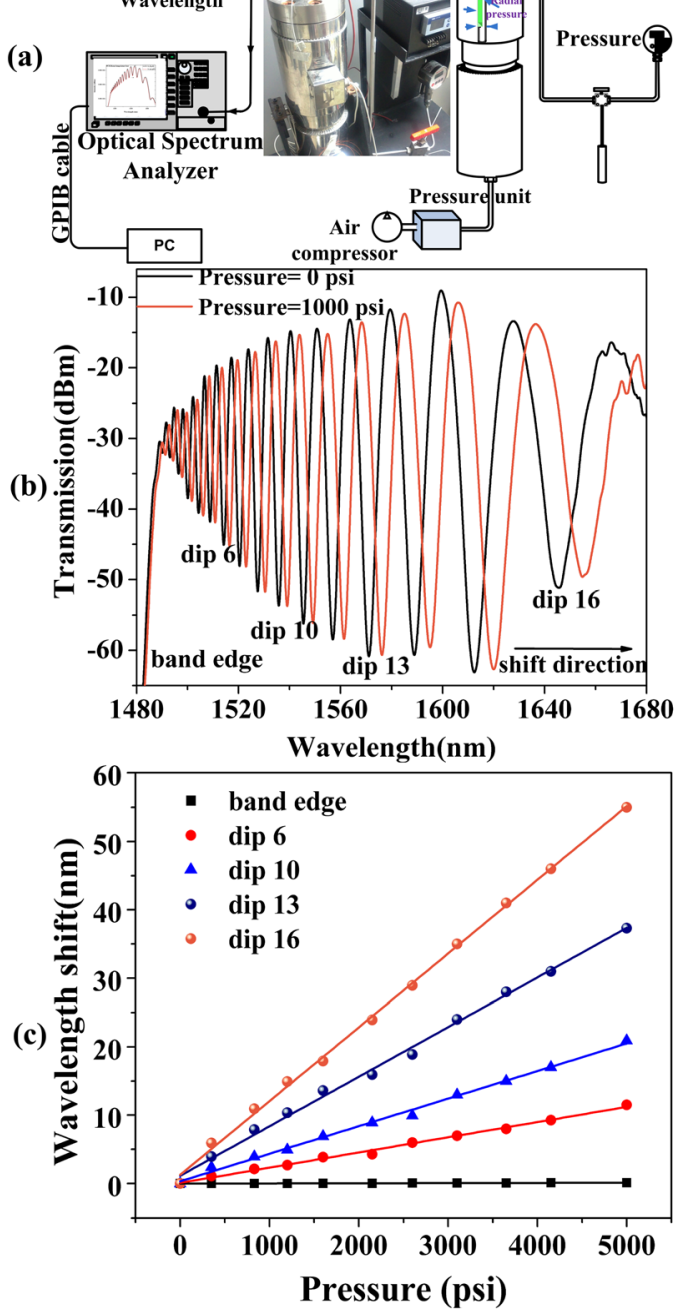

FIG. 2. (a) The experimental setup of high pressure sensor in the sagnac configuration. (b) The shift direction of spectral normalized transmission for two pressures 0 psi and 1000 psi. (c) The sensitivity of five different wavelengths under 5000 psi pressure. the range of $5 \times 10^{-5} \mathrm{~nm} / \mathrm{psi}$ at the band edge, as insensitive wavelength, to $1.3 \times 10^{-3} \mathrm{~nm} / \mathrm{psi}$ at $1680 \mathrm{~nm}$, as a highly sensitive wavelength. In Figs. 2(b) and 2(c), we summarized our results which show the shift direction of the spectral transmission of two different pressures 0 psi and $1000 \mathrm{psi}$ and the sensitivity of five different wavelengths under 5000 psi radial pressure, respectively. Clearly, the shift and sensitivity are the linear function of the applied pressure and are additive in the wavelength interval. To understand the impact of radial pressure on different wavelengths changes, we simulated our HC-PCF by coupling $\mathrm{RF}^{14}$ and Mechanics modules of commercial software (Comsol Multiphysics). By this two coupled modules, we were able to determine the resulting split of two polarizations of the fundamental core modes (FMs) and mode coupling between FMs and surface modes (SMs) under radial pressure.

As the first step of the simulation and based on the theory of elasticity, inhomogeneous unit cells (pentagons and hexagons) have a distinctive and exclusive effective elastic modulus $\left(E_{e f f}^{r, z}\right)$ in the radial (r) direction. According to Eqs. (2) and (3), the radial direction Young's modulus of the innermost ring is a function of air-filling ratio $(\eta)$ while Poisson's ratio is constant ${ }^{15,16}$

$$
E^{r}{ }_{e f f}=\frac{3}{2}(1-\eta)^{3} E_{m},
$$

and

$$
v_{r}=v_{0} .
$$

Here, $\mathrm{E}_{\mathrm{m}}$ is the Young's modulus of the silica material. The value of effective parameters for the honey comb inner cladding is listed in Table I.

Using this table and the geometrical parameters shown in Figs. 1(b) and 1(c), also according to the theory of elasticity in cellular solids, we calculated the deformation and displacement of each cell. In this step of simulations, by using the mechanics modules, we investigated the deformation of thin silica walls of hexagonal cells compared with thick silica walls of pentagonal ones under radial pressure.

TABLE I. The value of effective parameters $\eta$ and $\mathrm{E}_{\text {eff }}^{\mathrm{r}}$ of the honey comb inner cladding.

\begin{tabular}{lcc}
\hline \hline Region & $\mathrm{H}(\%)$ & $\mathrm{E}_{\text {eff }}^{\mathrm{r}}$ \\
\hline Pentagons of the innermost ring & 78 & $1.15 \mathrm{GPa}$ \\
Hexagons of the innermost ring & 96 & $7 \mathrm{MPa}$ \\
Five rings of similar hexagons & 85 & $365 \mathrm{MPa}$ \\
\hline \hline
\end{tabular}




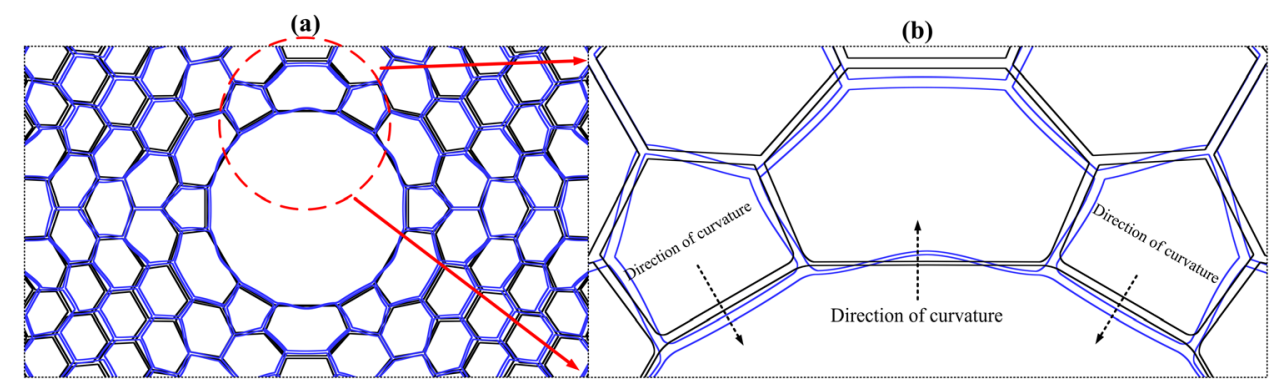

FIG. 3. (a) The deformation of HCPCF under the radial pressure. (b) The deflection of the innermost wall of hexagonal and pentagonal cells under radial pressure.

The distributed load under external radial force within the hexagon net will be transferred to the inner nodal points which were joined to the adjacent nodes by the thin silica walls. In this point, the length and thickness of the silica walls and also the wall angles of nodal points are the main factors. In a good assumption, the innermost walls of hexagonal and pentagonal cells around the air core have beam roles. According to the theory of elasticity or beam bending theory, a beam with narrow rectangular cross section and circular axis is constrained and bent by a force applied at the two ends. Deformation and bending moment at any point of beam are proportional to the length of the cell wall and angle in the nodal points and inversely proportional to the thickness of the walls. ${ }^{16,17}$ According to the boundary conditions, the outer and inner boundaries of innermost walls are free from external forces and the nodal points are under the same pressure. As Fig. 3 shows, when one cell is sheared, all its walls will bend. The innermost wall of hexagonal cells becomes an outward convex while the wall of pentagonal ones is curved inward concavely visualized with arrow marks in Fig. 3(b).

In the second set of simulations, the mode analysis module is coupled to the mechanics modules. These simulations allow us to elicit more details about crossing event occurring between the two polarizations of the fundamental core modes. ${ }^{18,19}$ According to the simulations, intensity expansion of core modes (FMs) in the slow axis direction (FM1) is more than that of the fast one (FM2). Therefore, once the innermost ring is deformed, the intensity expansion of the core modes in the slow axis will be affected more than that of the fast axis. Furthermore, the effective mode index of confined surface modes in the innermost walls of hexagonal cells (SM2) will decrease more than other ones (SM1).

The innermost walls have overlapping domain roles, where the core modes and surface modes interact with each other. In other words, the energy transfer between the two fundamental air core modes is possible only through the silica walls. To understand the actual interaction between the core modes and the surface modes which occurs during exerting pressure, we examined the behavior of these modes under radial pressure. Fig. 4(b) shows the dispersion curves of the two linear polarizations of fundamental modes (FM1 and FM2) and the two surface modes (SM1 and SM2). The evaluation of the effective mode index ( $\left.\mathrm{n}_{\mathrm{eff}}\right)$ before and after exerting pressure unveils the distinguishable behaviors of two polarizations in the vicinity of band edge at $1480 \mathrm{~nm}$ near avoided crossing region at $1680 \mathrm{~nm}$.

According to Fig. 4(c), the dashed-dotted red lines show that exerting pressure leads to more separation in effective index FM1 in comparison with FM2, especially in the vicinity of band edge at $1480 \mathrm{~nm}$. This means that $\left|\Delta n_{\text {eff }(F M 1)}\right|>$ $\left|\Delta n_{\text {eff }(F M 2)}\right|$ that $\Delta n_{\text {eff }}=n_{\text {eff }}\left(P_{1}\right)-n_{\text {eff }}\left(P_{0}\right)$. The group birefringence of simulation can be expressed as

$$
B_{g-\operatorname{sim}}=n_{\text {eff }(F M 1)}-n_{\text {eff }(F M 2)} .
$$

Here, $B_{g-s i m}$ is the group birefringence of simulation result and $n_{\text {eff }}\left(P_{1}\right), n_{\text {eff }}\left(P_{0}\right)$ are effective modes index after and before exerting pressure, respectively; one of our main results is decreasing the effective mode index in the wavelength intervals. In spite of increasing the amount of (a)

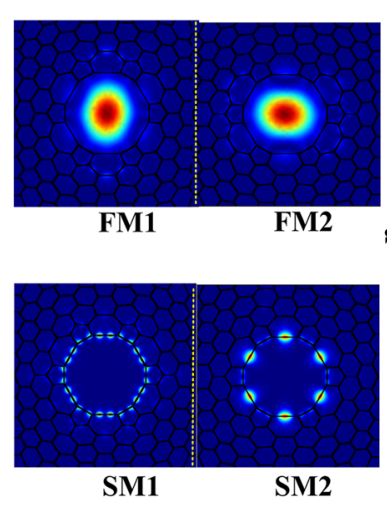

(b)

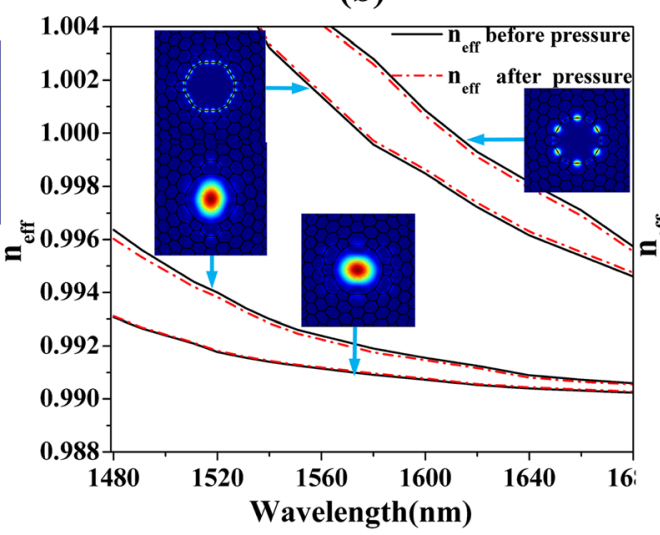

(c)

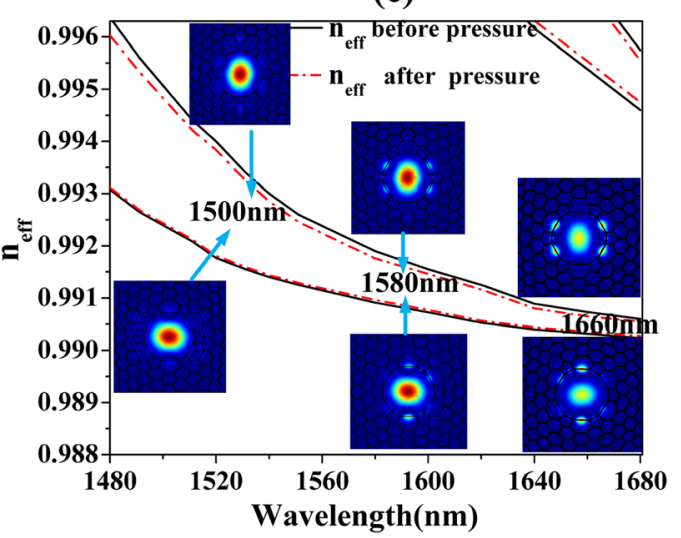

FIG. 4. (a) The intensity distributions of different modes, two fundamental modes (FM1, FM2) and two surface modes (SM1, SM2). (b) The variation of the effective mode index of different modes (black lines are $\mathrm{n}_{\text {eff }}$ before exerting 1000 psi pressure and dashed-dotted red lines are $\mathrm{n}_{\text {eff }}$ after exerting 1000 psi pressure). (c) The variation of the effective mode index of two FMs (the spectral transformation of two FMs for three wavelengths: 1500 nm, 1580 nm, and $1660 \mathrm{~nm})$. 


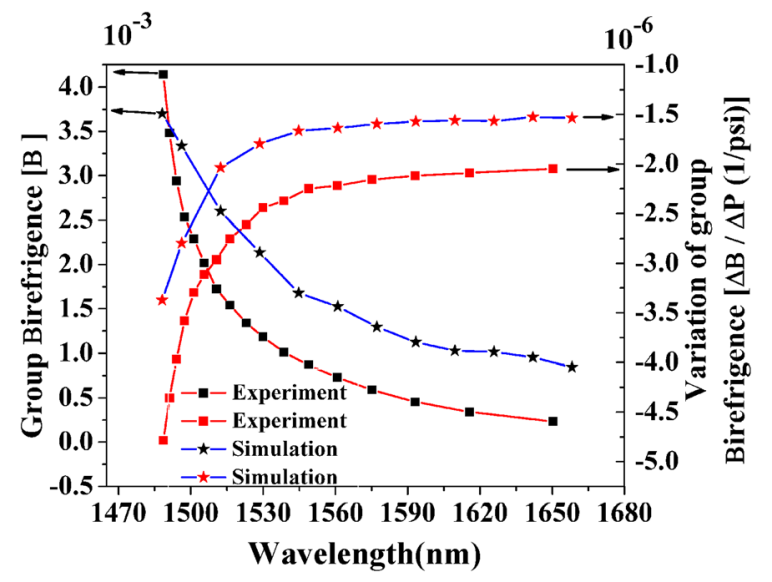

FIG. 5. The experimental (red lines) and simulation (blue lines) results of group birefringence (left) and variation of group birefringence (right) under 1000 psi pressure.

$n_{\text {eff(FM2), }}$ the amount of $n_{\text {eff(FM1) }}$ decreases which brings about a decreasing behavior in the total effective mode index. The other result is the variation of intensity expansions of two FMs obtained for wavelengths as shown in Fig. 4(c) for three wavelengths: $1500 \mathrm{~nm}, 1580 \mathrm{~nm}$, and $1660 \mathrm{~nm}$. The major interaction between all types of modes close to the avoided crossing region at $1680 \mathrm{~nm}$ causes a significant energy coupling in this region. Moreover, this coupling decreases the intensity expansion of FMs and, conversely, increases the intensity expansion of modes around the FMs. This interaction leads to a significant energy coupling between FM1 and FM2 and crossing event occurs by SMs in energy transmitter role. The energy coupling in this region has an important consequence known as the extreme sensitivity of this region to structural perturbation.

In order to measure the $B_{g-\exp }$, we measured the wavelength spacing $(\Delta \lambda)$ between both adjacent dips. It was observed that the wavelength spacing increased with wavelength increasing, from $2.9 \mathrm{~nm}$ to $33 \mathrm{~nm}$. Based upon the results of Eq. (1), the birefringence between both adjacent dips decreased with wavelength increasing. We obtained the group birefringence from $4.3 \times 10^{-3}$, at $1480 \mathrm{~nm}$, to $5 \times 10^{-4}$, at $1680 \mathrm{~nm}$. The simulation and experimental results of group birefringence are shown in Fig. 5.

The main results of experimental data analysis and simulations show that regardless of what we observed in the sensitivity of different wavelengths, the dispersion variation of wavelengths closer to the band edge is smaller than that of the wavelengths far away from the band edge. This dispersion variation indicates the major role of crossing event in energy transfer, near the avoided crossing region and away from the band edge. The simulation and experimental results of group birefringence variation showed exponential decay behaviors of the dispersion variation $\left(\left(B_{P 1}\right.\right.$ $\left.-B_{P 0}\right) / \Delta P$ ). These results with negative value determined from $-3.4 \times 10^{-6} 1 / \mathrm{psi}$ to $-1.3 \times 10^{-6} 1 / \mathrm{psi}$ and from
$-5 \times 10^{-6} 1 / \mathrm{psi}$ to $-1.8 \times 10^{-6} 1 / \mathrm{psi}$, respectively, which corroborated each other and are illustrated in Fig. 5.

In summary, behavior of dodecahedron HC-PCF fiber as a sensing head for high pressure measurements at different wavelengths under high radial pressure has been studied. The experimental results showed that the sensitivity of different wavelengths is linear function of the applied pressure and is additive in the wavelength interval. These results are from $5 \times 10^{-5} \mathrm{~nm} / \mathrm{psi}$ at insensitive wavelengths $(1480 \mathrm{~nm})$ to $1.3 \times 10^{-3} \mathrm{~nm} / \mathrm{psi}$ at high sensitive wavelengths $(1680 \mathrm{~nm})$. Moreover, the simulation and experimental results showed the exponential decay behaviors of dispersion variation from $-3.4 \times 10^{-6} 1 / \mathrm{psi}$ to $-1.3 \times 10^{-6} 1 / \mathrm{psi}$ and from $-5 \times 10^{-6}$ $1 / \mathrm{psi}$ to $-1.8 \times 10^{-6} 1 / \mathrm{psi}$ under pressure, respectively. According to the second set of the simulation results, due to the presence of the SMs around the FMs, the major interaction between these two kinds of modes in the avoided crossing region causes a significant energy coupling in this region. This interaction leads to a significant energy coupling between FM1 and FM2. The amount of wavelength shift or high sensitivity can be strongly affected by the energy transfer or crossing event in this region. Consequently, all the results clarify the role of the shape of the innermost ring such as core ellipticity, length of the cell walls, angles of nodal points, and thickness of the walls.

${ }^{1}$ A. W. Snyder and J. D. Love, Optical Waveguide Theory (Chapman and Hall, New York, 1983).

${ }^{2}$ J. C. Knight, J. Broeng, T. A. Birks, and P. St. J. Russell, Science 282, 1476 (1998).

${ }^{3}$ R. F. Cregan, B. J. Mangan, J. C. Knight, T. A. Birks, P. St. J. Russell, P. J. Roberts, and D. C. Allan, Science 285, 1537 (1999).

${ }^{4}$ P. St. J. Russell, Science 299, 358 (2003).

${ }^{5}$ J. A. West, Ch. M. Smith, N. F. Borrelli, D. C. Allan, and K. W. Koch, Opt. Express 12, 1485 (2004).

${ }^{6}$ S. Smolka, M. Barth, and O. Benson, Opt. Express 15, 12783 (2007).

${ }^{7}$ J. Villatoro, V. Finazzi, V. P. Minkovich, V. Pruneri, and G. Badenes, Appl. Phys. Lett. 91, 091109 (2007).

${ }^{8}$ S. H. Aref, R. Amezcua-Correa, J. P. Carvalho, O. Frazão, P. Caldas, J. L. Santos, F. M. Araújo, H. Latifi, F. Farahi, L. A. Ferreira, and J. C. Knight, Opt. Express 17, 18669 (2009).

${ }^{9}$ M. Pang and W. Jin, Opt. Express 17, 11088 (2009).

${ }^{10}$ S. O. Konorov, L. A. Mel'nikov, A. A. Ivanov, M. V. Alfimov, A. V. Shcherbakov, and A. M. Zheltikov, Laser Phys. Lett. 2, 366 (2005).

${ }^{11}$ X. Chen, M. J. Li, N. Venkataraman, M. T. Gallagher, W. A. Wood, A. M. Crowley, J. P. Carberry, L. A. Zenteno, and K. W. Koch, Opt. Express 12, 3888 (2004).

${ }^{12}$ P. J. Roberts, D. P. Williams, H. Sabert, B. J. Mangan, D. M. Bird, T. A. Birks, J. C. Knight, and P. St. J. Russell, Opt. Express 14, 7329 (2006).

${ }^{13}$ S. H. Aref, M. I. Zibaii, M. Kheiri, H. Porbeyram, H. Latifi, O. Frazão, F. M. Araújo, L. A. Ferreira, J. L. Santos, Jens Kobelke, and Kay Schuster, Opt. Commun 285, 269 (2012).

${ }^{14}$ R. Buczynski, D. Pysz, R. Stepien, A. J. Waddie, I. Kujawa, R. Kasztelanic, M. Franczyk, and M. R. Taghizadeh, Laser Phys. Lett. 8, 443 (2011).

${ }^{15}$ R. M. Christensen, Int. J. Solids Struct. 37, 93 (2000).

${ }^{16}$ L. J. Gibson and M. F. Ashby, Cellular Solids-Structure and Properties, 2nd ed. (Cambridge University Press, New York, 1997).

${ }^{17}$ S. P. Timoshenko and J. Goodier, Theory of Elasticity (McGraw-Hill, New York, 1970).

${ }^{18}$ K. Saitoh and M. Koshiba, Opt. Express 11, 3100 (2003).

${ }^{19}$ F. Poletti, N. G. R. Broderick, and D. J. Richardson, Opt. Express 13, 9115 (2005). 